\title{
Fluorescence-Guided Surgical Debridement of Chronic Osteomyelitis Utilizing Doxycycline Bone Labeling: A Technical Trick Revived
}

Garrhett G. Via, MD'; David A. Brueggeman, MD¹; Grant M. Slack, BS²; James M. Danias, DO; Jennifer L. Jerele, MD'

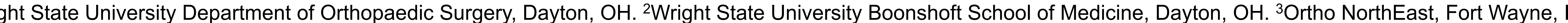
Boonshoft School of Medicine, Medical Student Research Symposium, Dayton, OH, April 2021

\section{Introduction:}

- Recalcitrant infections of bone and soft tissue are commonly encountered by orthopaedic surgeons.

Treatment strategies include long-term antibiotic administration for suppression and/or surgical debridement to obtain source control. ${ }^{1}$

- Intraoperatively identifying living, viable bone can be challenging

Debridement to bleeding bone may not correlate with a bone's metabolic state. ${ }^{8-10}$

Living bone is known to contain fluorophores (Tryptoph Tyrosine, Phenylalanine), which enable it to naturally

fluoresce.., 6

Bone Fluorescence is also achieved via the chelation of tetracycline molecules within the inorganic mineral matrix

- The quantity of healthy osteocytes correlates with visible fuorescence. Thus, fluorescence can be used to

demarcate viable bone. ${ }^{11-13}$

A frm of this technique was originally described in 2002 and has not been presented since that time.

\section{Surgical Technique:}

- Pre-op use of tetracycline allowing enough time for the metabolite to be incorporated into live bone.

- Doxycycline dosing can range from an IV formulation of $100 \mathrm{mg}$ delivered in pre-op holding to a multi-week

regimen in an oral formulation of $100 \mathrm{mg} \mathrm{BID}$.

- $\mathrm{R}$ lights turned off and a non-sterile team member uses a blacklight to illuminate the surgical field.

- The intensity of fluorescence will be in stark contrast to

any non-viable a

Operations including ORIF, IM nailing, placement of antibiotic-laden cement/beads into a bone defect to stage er clinical scenario.

\section{Case Series:}

- Intraoperative sample photos are included depicting bone fluorescence.

- Figure 1

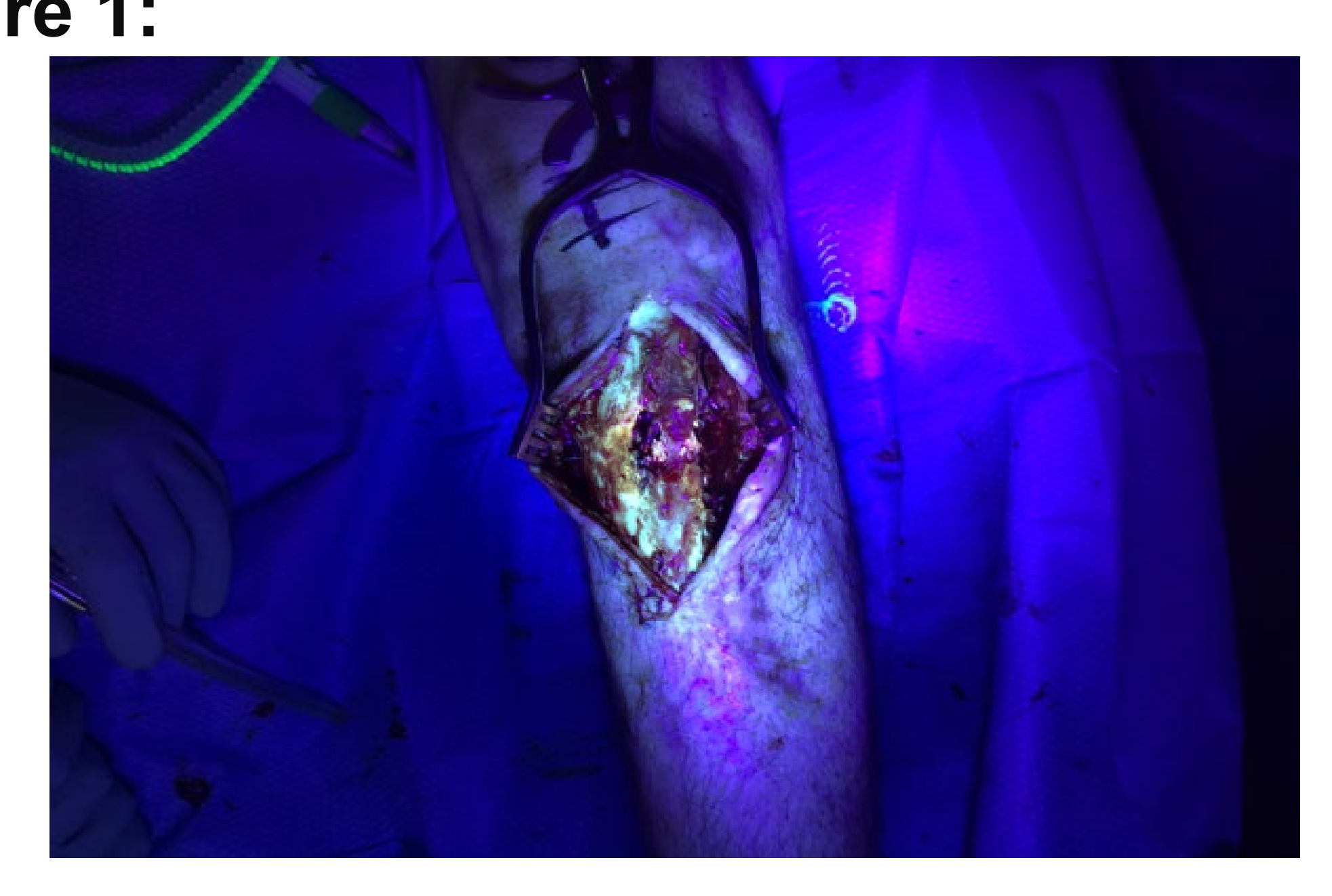

A) Intraoperative fluorescence of tibia noted pre-debridement

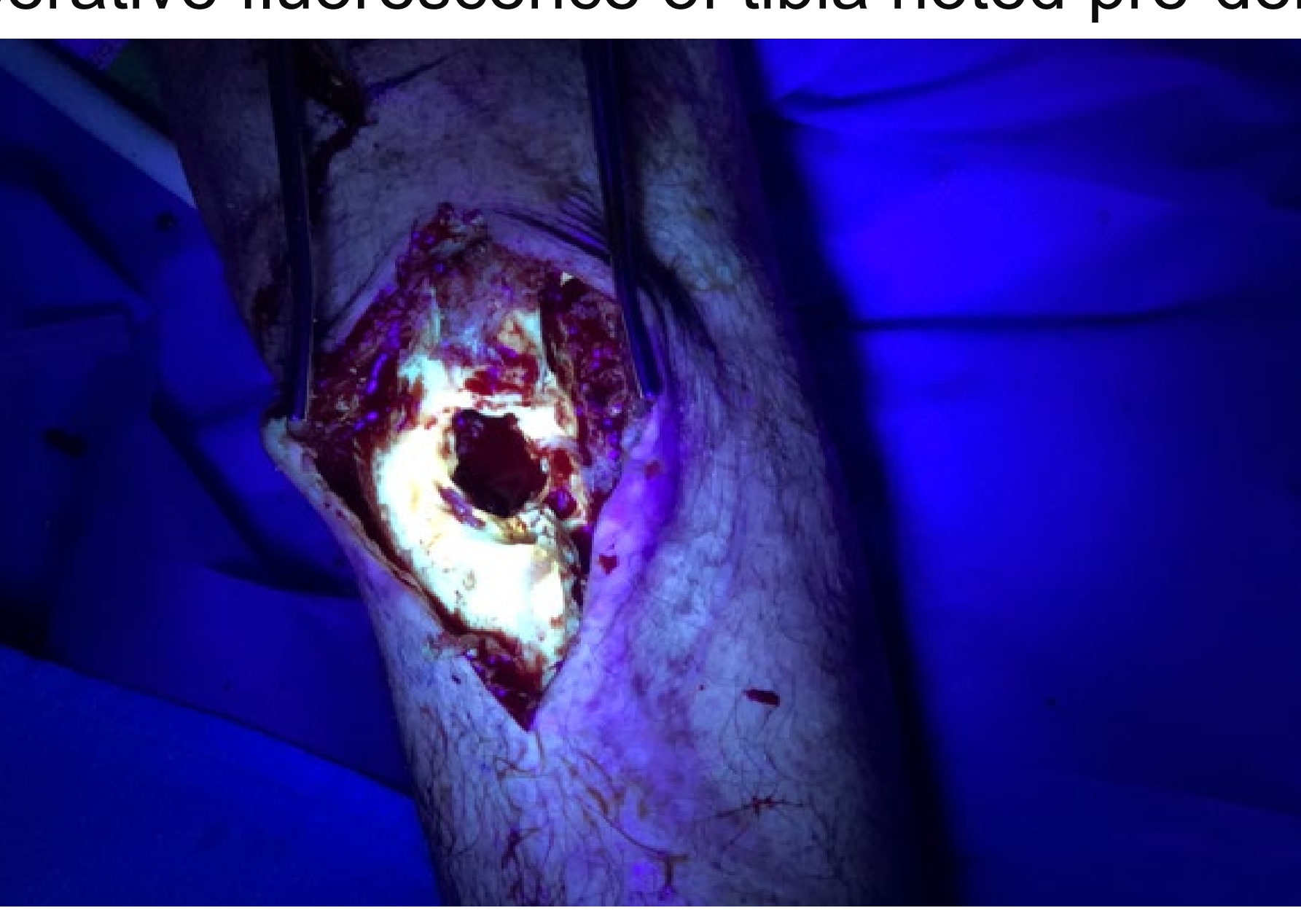

B) More complete fluorescence of the same tibia noted following completion of debridemen

- Figure 2:

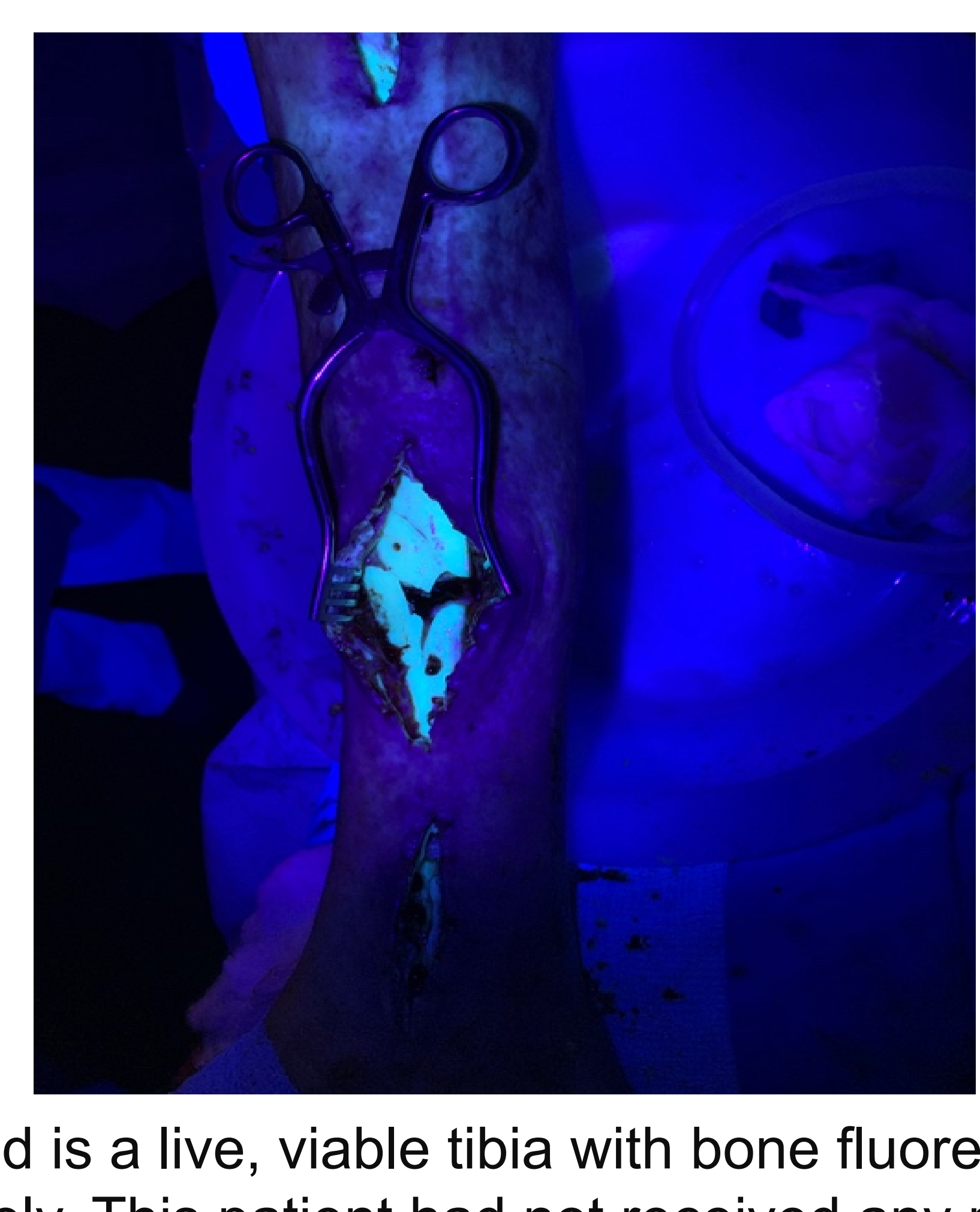

Demonstrated is a live, viable tibia with bone fluorescence noted doxycycline.
Case Series Continued

- Figure 3:

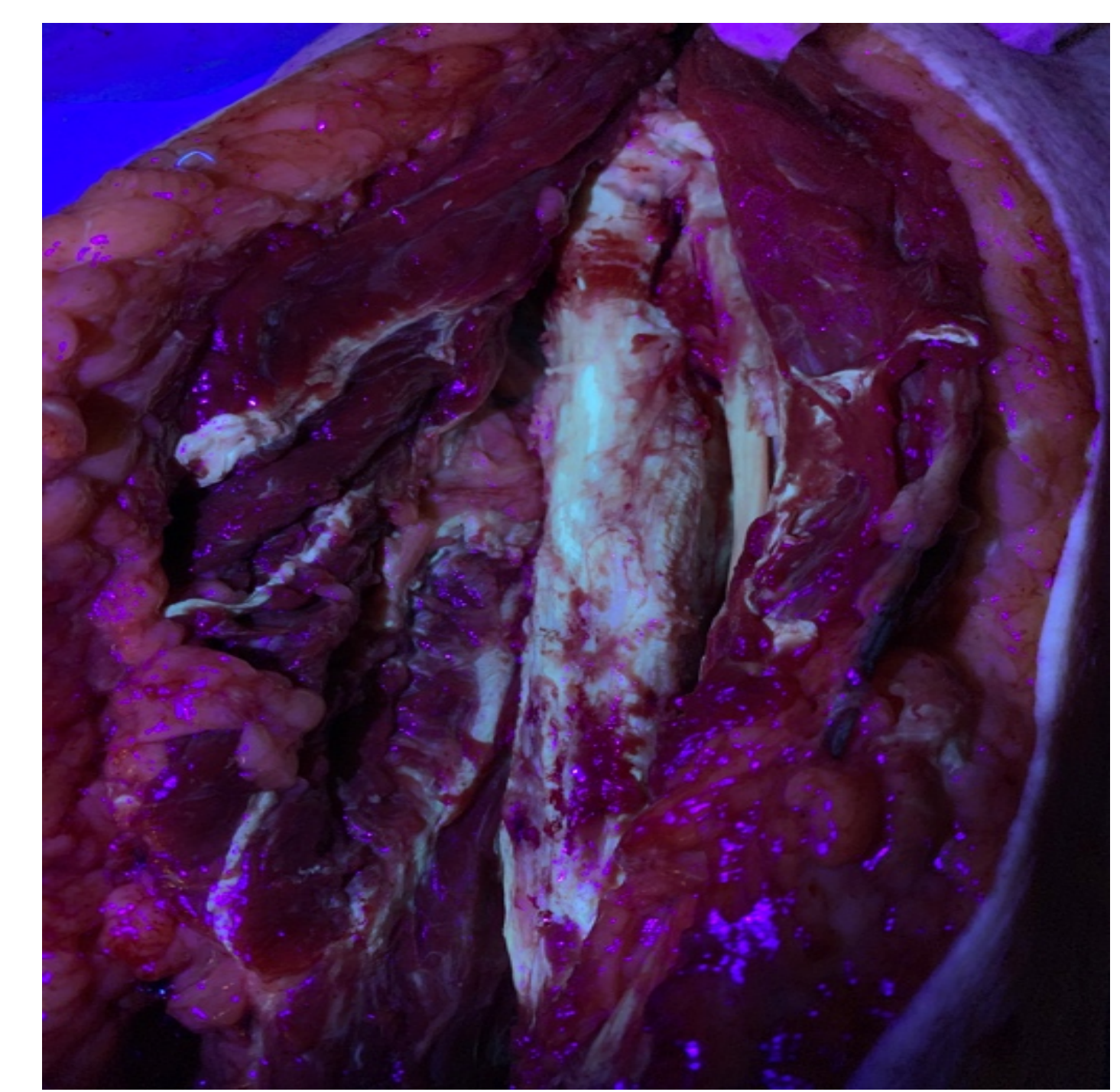

Specimen of fresh-frozen cadaveric humerus with blacklig exposure. Notice that minimal to no fluorescence is

Case Example: 30 YO F

- PMH: Recurrent skin abscesses.

- Presented in 2018 with vague leg pain.

- $\mathrm{CT}$ and MRI findings consistent with chroni osteomyelitis and intramedullary abscess of the tibia.

Taken to the OR for I\&D and cultures, which grew MRSA.

5 days later, taken back to the OR for

gentamicin/vancomycin-coated intramedullary nail and gentamicin/vancomycin-impregnated cement placement.

She was discharged on IV daptomycin and PO doxycycline 100mg BID.

7 weeks later returned for repeat I\&D with bone grafting of her tibia.

- Intraoperatively, OR lights were shut off and a black light was used to illuminate the wound.

All exposed bone fluoresced, indicating live bone with no need for further debridement or resection. At 1 year follow up, there was no tenderness over the tibia with full ROM and no complaints.

\section{Discussion:}

The senior author of this manuscript prefers preoperative administration of $100 \mathrm{mg}$ BID doxycycline for at least 1 month combined with the usage of an ordinary blacklight as the intraoperative exclation source.

- The use of an ordinary blacklight is cost-effective, readily attainable, and proven to be efficacious.

- A Imitation of the work is that lack of quantitative fluorescence measurements. It is hoped that any (t) to demonstrate its worth.

More formal trials to objectively quantify fluorescence intensity should follow.

\section{Conclusion:}

- The present manuscript has revived the concept of fluorescence-guided bone debridement based upon the bioch
tetracyclines

- Preoperative administration of doxycycline in a patient with osteomyelitis may allow for increased efficacy in guiding the surgical debridement procedures.

Variations in the technique do exist and can function quite well, however the concept of bone labelling for fluorescent-guided resection of chronic 列

challenging cases.

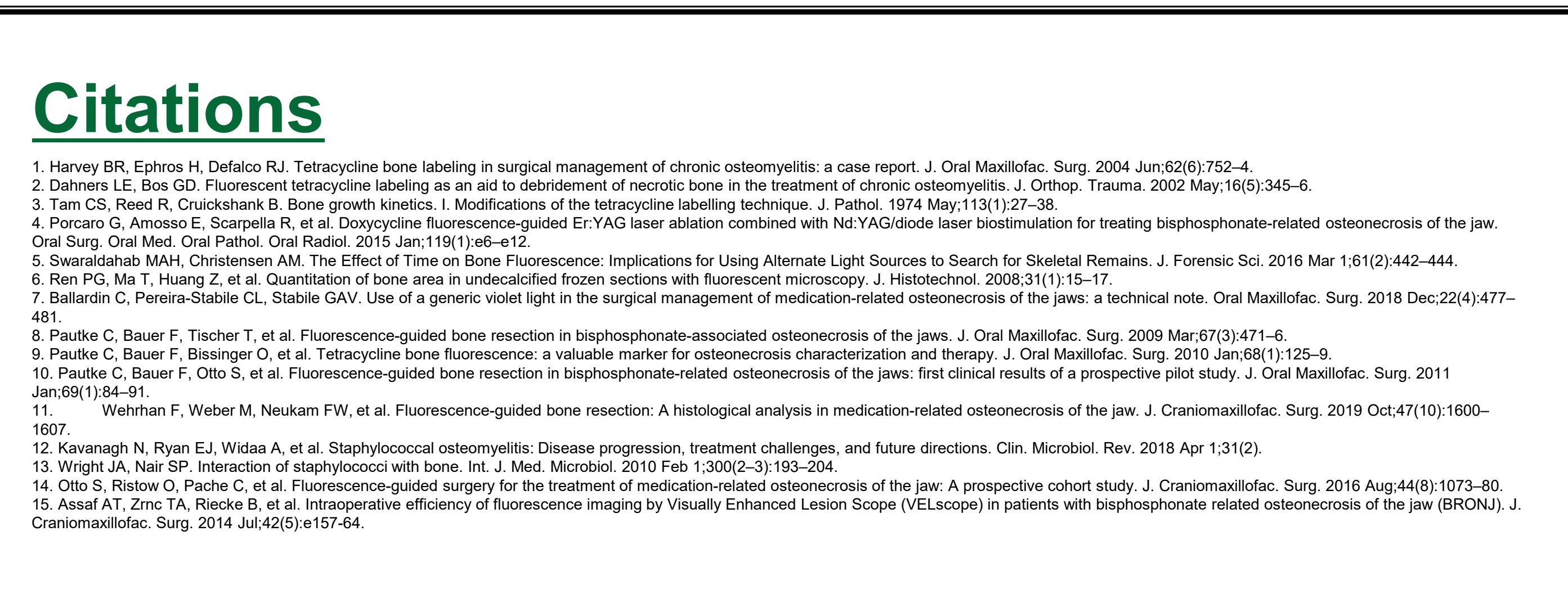

